\title{
RESEÑAS
}




\title{
UNIVERSIDAD PEDAGOGICA NACIONAL \\ Centro de Investigaciones
}

\section{LA ESCUELA PRIMARIA EN COLOMBIA}

\section{Araceli de Tezanos de Mañana}

\author{
Bogotá, enero 1981
}

\section{INTRODUCCION}

En los últimos años se han realizado en el país numerosas investigaciones que intentan explicar y describir la situación del sistema educativo colombiano.

Estos estudios parten de distintos enfoques del problema: algunos provienen de la Sociología, otros de la Economía, otros de la Psicología...

Los trabajos que se orientan desde la Sociología y la Economía tienden a explicar el sistema educativo en un sentido macro. Los primeros tratan de establecer las relaciones entre estructura social y su reproducción en el sistema educativo, a través de la incidencia de la estratificación de clases en la igualdad de oportunidades constitucionalmente establecida. Las investigaciones orientadas por la Economía tratan de establecer relaciones causales entre insumos y productos para establecer el grado de eficiencia del sistema educativo. Por otra parte, los estudios provenientes de la Psicología intentan explicar la situación educativa a través de una mayor relevancia de la especificidad del sujeto en los procesos de enseñanza-aprendizaje, intentando generalizaciones que hacen referencia sobre todo a los problemas de aprendizaje.

Muchas de estas investigaciones han tenido su repercusión en la elaboración e implementación de las políticas educativas nacionales. Pero en el campo específicamente pedagógico las innovaciones que se han puesto en marcha, en casi ninguno de los casos han partido de resultados o procesos de investigación en esta área.

Las orientaciones pedagógicas provenientes de la tecnología educativa que se han impuesto en los últimos años, a partir de modelos provenientes de los Estados Unidos, han sido meras replicaciones o traducciones de dichos modelos, mediante procesos de capacitación docente; pero no se ha investigado sobre los modos de adecuación de dichos métodos de enseñanza a la realidad nacional. Hasta la fecha se carece, por ejemplo, de una evaluación del modelo INEM, proveniente de las Universidades de Tallahasee y New México. Pensamos que esta evaluación, podría en cierta medida, sustituir la necesaria investigación previa.

A pesar de ello, se intenta implementar dicho modelo tecnológico a nivel de la escuela primaria, a través de las nuevas orientaciones curriculares, careciendo otra vez de datos o resultados de investigaciones sobre la realidad cotidiana de la escuela, para una previa 
identificación de problemas, a los cuales en sus aspectos académicos el nuevo currículo podría ofrecer alternativas de solución.

Sin embargo, es necesario hacer notar aquí, que sí existen en el país excelentes trabajos sobre los maestros, tanto desde el punto de vista de su formación (Briones, G. 1978), como desde el punto de vista de su situación socia] (Parra, R. y otros, 1980). Asimismo, es importante tener en cuenta el proyecto que sobre "La Historia de la Práctica Pedagógica en Colombia" se viene desarrollando en un trabajo conjunto de distintas instituciones universitarias $^{193}$. Este trabajo servirá sin duda, de marco obligatorio a las investigaciones pedagógicas que se realicen de aquí en adelante en el país.

Dentro de este contexto surge el proyecto "La Escuela Primaria en Colombia", que integra un estudio multinacional sobre "Escuela y Comunidad en América Latina", en el cual participan, inicial-mente, además de nuestro país, Bolivia y Venezuela.

\section{EL PROBLEMA}

Parece innecesario insistir sobre la importancia de la escuela primaria en nuestra sociedad, como pilar básico del sistema educativo.

A comienzos del Siglo XX la tradición sociológica y pedagógica francesa ${ }^{194}$ definió con claridad el papel de la escuela elemental como transmisora de los conocimientos que la sociedad donde está inserta acepta como válidos. Estos conocimientos hacen específica referencia a las nociones básicas de cálculo (sumar, restar, multiplicar, dividir) y al aprendizaje de la lectura y escritura. Sumado a ello, y como su consecuencia histórica inmediata, la escuela aparece como la mediadora específica de los procesos de socialización para el trabajo dentro del sistema productivo de la sociedad de la cual forma parte y a la que reproduce ${ }^{195}$.

La pregunta que surge inmediatamente es: ¿cómo la escuela lleva a cabo su función mediadora?

Desde Comenio a nuestros días, esta pregunta ha sido preocupación fundamental de la historia de las ideas y la práctica pedagógica; aunque, por otra parte, han habido respuestas fragmentarias sobre el problema que aquí se plantea. Algunas de ellas provienen de la Psicología de orientación conductista, cuyos trabajos de investigación han servido como marco de referencia a las corrientes tecnológicas en educación y han llevado a considerar la Pedagogía como un listado de normas y formulaciones técnicas, dejando por fuera toda reflexión teórica sobre el quehacer pedagógico, y provocando respuestas que ni explican, ni interpretan el problema de la medicación en su compleja totalidad $^{196}$.

Otras respuestas con una perspectiva más totalizante, para nuestra pregunta, provienen de la Sociología, pero en la mayoría de los casos resultan demasiado exterio-

\footnotetext{
193 "Historia de la Práctica Pedagógica en Colombia" — Proyecto inter-universitario (Un. de Antioquia, Un. del Valle, Un. Nacional, Un. Ped. Nacional) ZULOAGA, O.L. (Coordinador nacional) Medellín, 1980.

${ }^{194}$ DURKHEIM, Emile. “Educación como Socialización”. Ed. Sígueme, Salamanca, 1976.

${ }^{195}$ Las teorías sobre la escuela como reproductora del sistema socio-económico se han desarrollado en los trabajos de: Bordieu y Passeron - "La Reproduction" (Les editions de Minuit - París, 1970) Baudelot y Establet - "La escuela capitalista en Francia" (Siglo XXI Editores, México, 1975).

Bowles y Gintis - "Schooling in capitalist America" (N.Y. Basic Books, 1977) Michael Katz - "Class, Bureaucracy and Schools" (Praeger Publishers, N.Y., 1973).

${ }^{196}$ Estos métodos de enseñanza están descritos y analizados en los trabajos publicados por Skinner, Bloom, Gagné y Meyer.
} 
res a la institución pedagógica misma, puesto que parten de los supuestos teóricos antes mencionados de reflejo y reproducción.

Nosotros pensamos que el problema de la función mediadora de la escuela puede explicarse e interpretarse planteando una serie de preguntas directrices, tales como: ¿qué sucede en la escuela?, ¿qué se enseña?, ¿cómo se enseña?, ¿qué se aprende?, ¿por qué se aprueba? ¿por qué se fracasa?, ¿por qué se van los niños de la escuela antes de la finalización del ciclo escolar?, ¿por que se quedan?

Creemos que para encontrar una respuesta totalizante a estas preguntas directrices es necesario adentrarse en la institución escolar, que es la encargada por la sociedad de transmitir conocimientos y mediar procesos de socialización, a través de las relaciones que se establecen en su interior. Estas relaciones se reticulan entre los distintos grupos humanos que confluyen en la escuela: maestros, alumnos, padres. Los nexos que se establecen se centran en un interés por el conocimiento que se media por el trabajo, la comunicación y la afectividad. Estos elementos son las mediaciones de la relación pedagógica, que se establece institucional-mente.

El análisis de la relación pedagógica institucional ${ }^{197}$ permitirá, entonces intentar una respuesta global a las preguntas propuestas anteriormente.

Como el proyecto internacional del cual forma parte esta propuesta, tiene como objetivo último ver en qué medida y de qué manera el maestro contribuye al éxito o al fracaso de los niños en la escuela primaria, con las consecuencias que aparentemente tiene este último en los índices y tasas de deserción que presentan las estadísticas educativas, creemos necesario hacer algunas consideraciones y aclaraciones sobre los conceptos de "éxito" y "fracaso escolar", para clarificar el diseño de la investigación que presentaremos a continuación.

Debemos aclarar también que lo que expondremos a renglón seguido son anticipaciones hipotéticas sobre el tema, que deberán ser contrastadas durante el proceso reflexivo sobre el trabajo de campo.

Para nosotros el discurso sobre el "éxito y el fracaso escolar" debe ser considerado en tres niveles: a) la visión del Estado y el sistema educativo; b) la visión de los maestros; c) la visión que los padres, en los diferentes sectores sociales tiene sobre dichos conceptos. A esto se agrega la necesidad de establecer y analizar las posibles relaciones que existan entre estas tres visiones.

\section{a) La visión del Estado y el sistema educativo}

Es evidente que toda la legislación escolar del país tiende a establecer los mecanismos pertinentes para alcanzar altas tasas de retención en la escuela primaria, puesto que el Estado tiene muy claro el valor social y económico de esta educación inicial para el progreso y desarrollo de la sociedad.

El Estado colombiano hace gratuito y obligatorio este nivel de educación para toda la población según la Ley 39 de $1903^{198}$, con una duración de cinco años. Se agrega a esto

\footnotetext{
${ }^{197}$ Vale la pena aclarar que en este trabajo el concepto de institución no está restringido al aspecto físico de la escuela, sino que está considerado en un sentido socio histórico. Es en función de ellos que cuando hablamos de la escuela no se hace referencia a paredes o a cualquier otro tipo de límite físico.

${ }^{198}$ Ministerio de Educación Nacional - EDUCACION COLOMBIANA - Tomo I, Bogotá 1959 
una serie de decretos que castigan a través de multas a los padres que no cumplan con la obligación de enviar sus hijos a la escuela primaria.

Sin embargo, las estadísticas oficiales demuestran que hasta ahora no se han alcanzado los ideales propuestos por el acto legislativo ya que sobre cada 100 niños, el $32 \%{ }^{199}$ no alcanza a finalizar el ciclo educativo inicial, a pesar del alto porcentaje que el Estado invierte de su presupuesto de gastos para hacer cumplir los preceptos constitucionales según la legislación vigente ${ }^{200}$.

Podemos, en consecuencia, anticipar hipotéticamente que el Estado y el sistema educativo han fracasado en su aspiración de dar una educación inicial completa a todos los niños en edad escolar.

\section{b) Visión de los maestros}

Existen en la literatura pedagógica de los últimos quince años, definiciones muy claras sobre "el éxito y el fracaso escolar". Estas definiciones provienen del contexto teórico de las corrientes funcionalistas en educación. El niño etiquetado como "éxito escolar" es aquel que es capaz de alcanzar por lo menos el $80 \%$ de los objetivos que, entendidos como conductas observables terminales, se establecen en los programas escolares. El niño "fracaso", consecuentemente, es aquel que no alcanza el mencionado $80 \%$ de conductas observables terminales establecidas.

El maestro tiende entonces a resolver muy fácilmente el significado de estos conceptos: "éxito: el que puede", "fracaso: el que no puede".

A esto se agrega que en términos generales las razones o causas del "éxito" son interiores a la escuela y más específicamente aún, el salón de clase; en tanto, las razones del "fracaso" siempre son externas: deprivación económica o afectiva, mala nutrición, etc.

Existe, en consecuencia, una dualidad sobre las formas en que el maestro explica su incidencia en los logros de los niños.

Nuestra anticipación hipotética aquí sostiene que, el maestro siente la necesidad de la permanencia de los niños en la escuela durante todo el ciclo escolar legalmente establecido, pero, aparentemente, no ha reflexionado sobre el papel que él tiene en la posible permanencia de sus alumnos, en la escuela; y en muchos casos desconoce la visión que los padres de los niños tienen sobre la funcionalidad y utilidad de bis conocimientos escolares.

\section{c) Visión de los padres}

Existe una opinión con consenso social bastante general que afirma que "en la escuela se aprende a leer, a escribir y hacer cuentas". Todas estas habilidades son consideradas por los padres "como útiles para el trabajo"; en cambio no alcanzan, en términos generales, a ver la utilidad que pueden tener los conocimientos que los niños pueden adquirir sobre historia, geografía o ciencias naturales para su posterior o paralela inserción en el mercado laboral.

\footnotetext{
${ }^{199}$ DNP - UDS - Evolución del sistema educativo colombiano, 1964 - 1977, mayo, 1979, Bogotá.

${ }^{200}$ Constitución Política de la República de Colombia, Art. 41 (Art. 11, Plebiscito Diciembre 1o. de 1957)
} 
Los padres generalmente consideran que la escuela puede haber cumplido su función cuando sus hijos han adquirido las destrezas nombradas en primer lugar. La adquisición de estas prácticas no necesariamente dura los cinco años establecidos legalmente para el ciclo elemental del sistema educativo. Puede durar menos. En consecuencia, los padres pueden retirar a sus hijos de la escuela cuando ellos "mas o menos son capaces" de manejar una lectura mecánica y deletreada y las cuatro operaciones fundamentales del cálculo elemental.

En este momento para ellos la escuela ha cumplido con su función primordial: dar los instrumentos básicos para el trabajo.

A partir de esto podemos anticipar hipotéticamente que los padres no consideran "fracasos" a los niños que sin haber completado los cinco años de escolaridad primaria legalmente establecidos, son capaces de insertarse en el mercado de trabajo, aún en condiciones de sub-empleo, en razón de la edad de los niños.

\section{El diseño de la investigación}

Nuestro proyecto intenta hacer un análisis de la situación actual de la escuela primaria, pero no como un ente aislado, sino como un elemento que se articula dentro del todo social al cual está expresando y en muchas instancias legitimando. En consecuencia, en este estudio no se trata de ver "la calidad de la escuela en términos de experiencia interna o externa del sistema" o "los problemas de aprendizaje", sino la escuela como expresión de una totalidad social objetiva.

Esta totalidad social objetiva se concretiza para nosotros en la relación pedagógica institucional en la cual intervienen: maestros, niños y padres. Pero, también consideramos que esta relación pedagógica se transforma en su proceso histórico y adquiere dimensiones diferentes según los distintos con-textos. Por esta razón, el proyecto tiene a considerar los siguientes elementos institucionales: maestros, niños en edad escolar (dentro y fuera de la escuela) y padres. Asimismo, se tendrán en cuenta los siguientes contextos: a) urbano (grupos marginales y obreros); b) rural y c) semi-rural.

El esquema del estudio propuesto contiene los siguientes elementos:

\section{Contexto socio-económico (comunidad)}

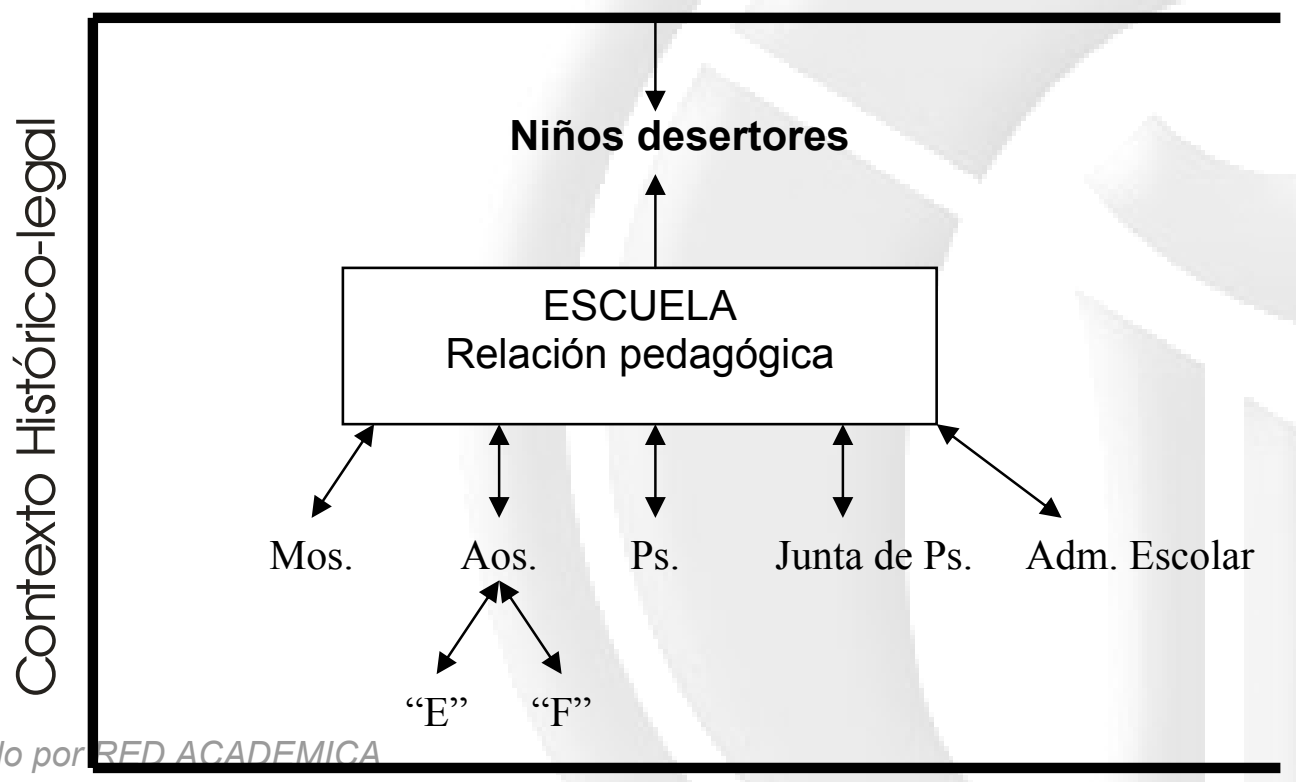




\section{Metodología del estudio}

La investigación aquí propuesta se enmarca dentro de una perspectiva etnográfica. Esto supone no solo la aplicación de cierto tipo de técnicas como la observación participantes, las entrevistas no estructuradas o el juego de roles, sino también una forma de interpretación y análisis de los datos recogidos a partir de la aplicación de las técnicas mencionadas y otras.

Creemos necesario, en consecuencia, hacer algunas consideraciones sobre esta perspectiva que ha adoptado nuestro-proyecto.

La metodología de la etnografía ha sido desarrollada y utilizada dentro de la Antropología. La etnografía ha sido definida de diversas maneras por aquellos antropólogos que han tratado de construir una herramienta, encontrar su esencia y desarrollarla en el tiempo.

Desde el estructuralismo-funcionalista se acepta que la etnografía es una tarea descriptiva en la cual el investigador intenta describir e interpretar la naturaleza del discurso cultural.

Para otras escuelas o corrientes antropológicas, la tarea descriptiva no puede ser un "reflejo" de la realidad concreta observada ${ }^{201}$, aunque se parte de registros lo más "objetivos" posibles de dicha realidad. Dichos registros constituyen "documentos etnográficos". Estos "documentos etnográficos" serán necesariamente leídos e interpretados por el investigador, tratando de descubrir "la red de significaciones" que según Geertz $^{202}$, constituye la cultura. La etnografía se define aquí como una relación entre teoría y método ${ }^{203}$.

Para ser capaz de realizar una buena etnografía, el investigador debe tener una teoría "razonable" de la sociedad para poder comprender su estructura y sus interacciones en la realidad y desde su propia práctica.

Un estudio etnográfico es necesariamente totalizante ("holistic") ya que la tarea del investigador es mostrar la relación entre su objeto de estudio (en este proyecto "La escuela primaria") y la totalidad social en la cual está articulado.

Se parte del supuesto que "la práctica de la etnografía permite descubrir el saber cultural que posee un grupo de personas así como también los modos en los cuales ese saber cultural es empleado en las interacciones sociales"204. Pero este descubrimiento no se hace desde la "tabula rasa" del investigador, pues éste llega a esa práctica con una historia, con teorías construidas, con visiones del mundo. En consecuencia, el producto final es esencialmente una síntesis de los dos modelos de la realidad social ${ }^{205}$ la del investigador y la del objeto de estudio.

\footnotetext{
${ }^{201}$ Rockwell, Elsie. "La relación entre etnografía y teoría en la Investigación Educativa”, DIE - IPN, México 1980 (mimeo)

${ }^{202}$ Geertz, C. "The interpretation of Culture," N.Y. Basic Books, 1973 -Cap. 1

${ }^{203}$ Herskovits, Melville - "Some problems•in Etnography," en "Method and Perspective in Anthropology," Robert F. Spencer ed. Minneapolis, University of Minnesota Press, 1954.

${ }^{204}$ Wilcox, Kathleen - "The etnography of schooling: implication s for educational policy-making," Project Report - Department of Human Development - California State University/Hayward, 1980

${ }^{205}$ Ogbu, John - "School Etnography: a multi-level approach.” Department of Anthropology, University of California, Berkeley, 1980 
La etnografía lleva necesariamente al conocimiento de una realidad concreta, que es histórica y está delimitada en el tiempo y el espacio.

La relación pedagógica institucional responde a las categorizaciones antedichas, y es en razón de ello que nuestro proyecto ha adoptado esta perspectiva.

\section{- Técnicas que se aplican en esta investigación}

Antes de reseñar las técnicas con que se trabaja en este proyecto, debemos aclarar que aunque la observación participantes es la técnica principal de recolección de datos, la flexibilidad metodológica es la característica fundamental de los procesos de investigación etnográficos.

- Realizaremos observaciones en los salones de clase durante el período comprendido entre el mes de febrero y junio de 1981, y retornaremos a este trabajo desde mediados de octubre hasta la finalización del año académico.

Dichas observaciones no son focalizadas, sino que se intenta "ver cada vez mas. Haremos especial hincapié en los registros lingüísticos textuales, pero también tendremos en cuenta las conductas no verbales tanto de niños como maestros.

- Llevaremos a cabo entrevistas no estructuradas con los maestros y directivas de las escuelas, así como con padres y niños.

-Al interior del salón de clase realizaremos sesiones de juego de roles con los niños para ver cómo ellos ven a sus compañeros y maestros.

- Realizaremos talleres con maestros, una vez finalizado nuestro primer período de observación, para hacer reflexiones sobre los registros de observación obtenidos.

—Elaboraremos historias de vida de niños considerados "desertores" por el sistema educativo, que ubicamos en nuestros lugares de trabajo.

—Elaboraremos historias de vida de niños que el maestro considera "éxitos" y "fracasos

\section{Los objetivos de la investigación}

Creemos que desde esta perspectiva podremos cumplir con el objetivo general que se propusiera inicialmente para este proyecto de investigación:

Examinar la situación de aprendizaje, analizando algunas escuelas primarias en Colombia, para encontrar por qué y cómo los maestros contribuyen al éxito o al fracaso de sus alumnos.

Pero el proyecto también pretende:

_ producir recomendaciones para una política de entrenamiento de maestros;

—analizar críticamente los métodos y técnicas aplicados al interior de esta investigación, para que puedan ser empleados por los maestros para la elaboración de diagnósticos, así como también para que se pueda hacer una reflexión crítica sobre su práctica pedagógica; 
- hacer una reflexión crítica que contraste los aspectos teóricos planteados en la investigación con la información relevada durante el trabajo de campo, para posibilitar el comienzo de la reconstrucción de una teoría pedagógica que permita desarrollos ulteriores del quehacer pedagógico acordes con nuestra realidad colombiana.

\section{LA IMAGEN DEL MAESTRO RURAL}

Los estudios sobre educación rural realizados por las diversas entidades y/o personas que se interesan por este tema en el medio colombiano, han abarcado hasta el momento aspectos imprescindibles para el conocimiento y el diagnóstico de los principales logros y problemas del servicio educativo ofrecido en las áreas rurales. Tal es el caso, por ejemplo, de los trabajos existentes sobre la cobertura del sistema y la evaluación del rendimiento escolar, las fuentes de financiación y la administración del presupuesto educativo, la formación y capacitación del personal docente, la legislación e historia de la educación rural, las técnicas y concepciones pedagógicas implementadas en el área, los inventarios de recursos didácticos y de infraestructura.

Aunque semejante diversidad de tópicos indican la existencia de un conocimiento bastante amplio sobre el estado actual de la educación rural, también es posible señalar que entre todos ellos, la dimensión social de la educación es quizá la más olvidada, por cuanto, en la mayoría de los casos, no se ha visto su utilidad práctica inmediata en términos de formulación de políticas o de asignación de recursos presupuéstales.

En efecto, son excepcionales los casos en los cuales los intereses de los investigadores en educación se han orientado hacia el análisis de aquellas situaciones y factores que en un momento dado permitirían una adecuada interpretación del significado dado a la educación, la escuela y el maestro en las comunidades a las cuales pretenden servir; y, por tanto, del tipo de relaciones e interacciones que es posible encontrar, y de las que sería deseable llegar a establecer entre todos los actores del proceso educativo en comunidades rurales. El interés de este proyecto es abrir una nueva línea de investigación en esta dirección a partir de un estudio que, por sus objetivos y dimensiones, sólo pretende explorar el tema.

El objetivo central del estudio es pues llegar a conocer, para el caso de dos escuelas rurales incompletas -que podrían considerarse típicas de las regiones campesinas colombianas - cuál es la imagen del maestro rural ante sí mismo, ante sus alumnos y ante la comunidad en la cual trabaja.

Por otra parte, como la categoría analítica Imagen del Maestro conlleva distintas dimensiones, también es propósito del estudio explorarlas sobre el terreno y re-definirlas si es necesario, establecer las posibles incongruencias y contradicciones existentes entre ellas e identificar las causas probables de las mismas.

Además, se ha procurado seleccionar las escuelas de manera tal que las dos pertenezcan a comunidades rurales con características sociales, económicas y culturales similares, con el propósito de neutralizar, hasta donde sea posible hacerlo, la influencia de factores ajenos a las condiciones específicas del docente en las diferencias que, como se prevé, podrán hallarse en las interacciones maestro-alumnos, maestro-comunidad. 